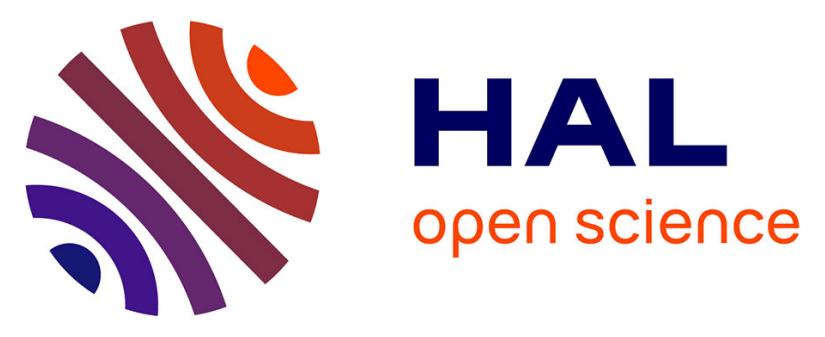

\title{
PICOLO: turbulence simulator for adaptive optics systems assessment in the context of ground-satellite optical links
}

\author{
Marie-Thérèse Velluet, Cyril Petit, Louis Le Leuch, Aurélie Montmerle \\ Bonnefois, Jean-Marc Conan, Frédéric Cassaing, Nicolas Védrenne
}

\section{To cite this version:}

Marie-Thérèse Velluet, Cyril Petit, Louis Le Leuch, Aurélie Montmerle Bonnefois, Jean-Marc Conan, et al.. PICOLO: turbulence simulator for adaptive optics systems assessment in the context of groundsatellite optical links. SPIE Remote Sensing 2020, Sep 2020, Washington (Online Only), United States. pp.1153207, 10.1117/12.2573954 . hal-03037592

\section{HAL Id: hal-03037592 https://hal.science/hal-03037592}

Submitted on 3 Dec 2020

HAL is a multi-disciplinary open access archive for the deposit and dissemination of scientific research documents, whether they are published or not. The documents may come from teaching and research institutions in France or abroad, or from public or private research centers.
L'archive ouverte pluridisciplinaire HAL, est destinée au dépôt et à la diffusion de documents scientifiques de niveau recherche, publiés ou non, émanant des établissements d'enseignement et de recherche français ou étrangers, des laboratoires publics ou privés. 


\title{
PICOLO: Turbulence simulator for AO systems assessment in the context of ground-satellite optical links
}

\author{
M.-T. Velluet* ${ }^{1}$, C. Petit, L. Le Leuch, A. Bonnefois, J.-M. Conan, F. Cassaing, N. Védrenne \\ Onera, The French Aerospace Lab, 29 av. de la Division Leclerc, F92322 Châtillon
}

\begin{abstract}
Optical communication between satellites and optical ground stations is a promising technology for high-speed data transfer between Space and Earth. However, the atmospheric turbulence present in the last few tens of kilometres near the ground has a significant impact on the quality of the link and reduces the transfer rate due to signal fading. To explore the robustness of adaptive optics (AO) systems to mitigate the turbulence effects at low elevation angles, we have developed a bench representative of the propagation channel between the ground and the satellite. The aim of the paper is to present the system analysis, the definition choices and the simulated performance that have conducted to the actual bench. We also describe the set-up and the characterisation of the main components.
\end{abstract}

Keywords: strong turbulence, optical telecommunication, adaptive optics, experimentation in labs

\section{INTRODUCTION}

Optical communication between satellites and optical ground stations (OGS) is a promising technology for high-speed data transfer between Space and Earth. However, the atmospheric turbulence present in the few tens of kilometres close to the ground has a great impact on the quality of the link and reduces the transfer rate due to signal fading. The use of adaptive optics helps to mitigate this effect, but unlike astronomical applications where the observation elevation angle is quite high, here we have to cope with low elevation angles, typically $20^{\circ}$ and $10^{\circ}$ if possible. In fact, in the context of telemetry (downloading high resolution images from space), the needs in terms of data throughput between a LEO satellite and the ground are increasing, the target is typically a few $\mathrm{Gb} / \mathrm{s}$. To transfer as much data as possible while the satellite is in view, we also have to increase the link duration, which means that data downloading should start at the lowest possible elevation (at least $10^{\circ}$ ). At these angles, the propagation distance in the atmosphere is very long $(>50$ $\mathrm{km}$ ) and scintillation effect is predominant making wavefront measurements more complex.

Moreover the satellite elevation evolving during the observation, we observe unstationary effects (mainly evolution of $r_{0}$ due to different propagation distances inside the atmosphere, mean flux quantity that can fluctuates from 1 to $10, \ldots$ ) which also affects the quality of the WaveFront (WF) measurements and the effectiveness of AO correction.

With the help of simulation, it is possible to develop and evaluate the performance of robust AO systems under such operating conditions. But field trials are necessary to test and validate this theoretical analysis, in particular the robustness of the system to strong scintillations and large flux fluctuations. However, the access time to LEO satellites is very limited both because of its visibility time and the fact that currently only a few LEOs are equipped with an on-board optical terminal. It is therefore imperative to test the equipment before conducting on-sky experiments. This is why we have developed a specific bench representative of an optical link between Space and Earth, this bench is called PICOLO.

A few benches have been developed and described in literature but they are designed for a specific task and do not deliver a turbulence-disturbed laser beam representative of the propagation between a satellite and the ground at low elevation integrating scintillation effects and unstationary turbulence effects. We can cite:

- Brady et al. [1]; the bench described is dedicated to the validation of an AO pre-compensation uplink. It is composed of a single phase screen. Moreover, it is not properly scaled because some effects are underestimated like the beam wandering.

* corresponding author: marie-therese.velluet@onera.fr 
- Kudielka et al. [2], the described bench is representative of an uplink at $30^{\circ}$ elevation, day-time conditions, the turbulence is sampled by two screens, the parameters are scaled. It integrates the AO system.

- Zhen Qu et al. [3]; Turbulence is simulated with 2 Spatial Light Modulators (SLM) used in reflexion in order to have at the end 4 different phase screens. It is representative of a horizontal application.

The paper is structured as follows. In the first part, the main phases of the bench definition are detailed, in particular the different steps followed to optimise and validate the sampling of the turbulence volume by only 3 layers and the downscaling of the experimental set-up to be representative of scintillation and phase effects. The second part is devoted to the integration and testing of the main elements of the bench. In the third part, possible evolutions of the bench to meet new needs are presented.

\section{BENCH DEFINITION}

\subsection{Experimental bench specification}

The bench must be representative of a link between a LEO satellite and the ground with an elevation angle of $10^{\circ}$. The propagation distance through turbulence is around $100 \mathrm{~km}$ that we want to reduce to a distance smaller than $2 \mathrm{~m}$. A downscaling of the main parameters needs to be performed, it means that:

- Turbulence profile is sampled on a low number of layers (typically 3);

- Diffraction and phase effects are preserved to guarantee the representativeness of the propagation channel;

- Temporal effects are also preserved in order to be able to simulate an AO system with a sampling frequency up to $2 \mathrm{kHz}$; the use of SLM to simulate the turbulence is not adapted in this case.

The bench has to be compact and movable, so the use of digital micro-mirror device (DMD) is not suitable either. For this reason, transmissive phase screens have been considered and mounted on a controlled rotating support to simulate the cross-wind speed with the correct scale factor. To produce non-stationnary turbulence near the ground (simulation of turbulence burst) we introduce a SLM in a conjugated plane of the entrance pupil. Because this effect is slower than "regular" turbulence the use of SLM is possible.

\subsection{Definition of the phase screens}
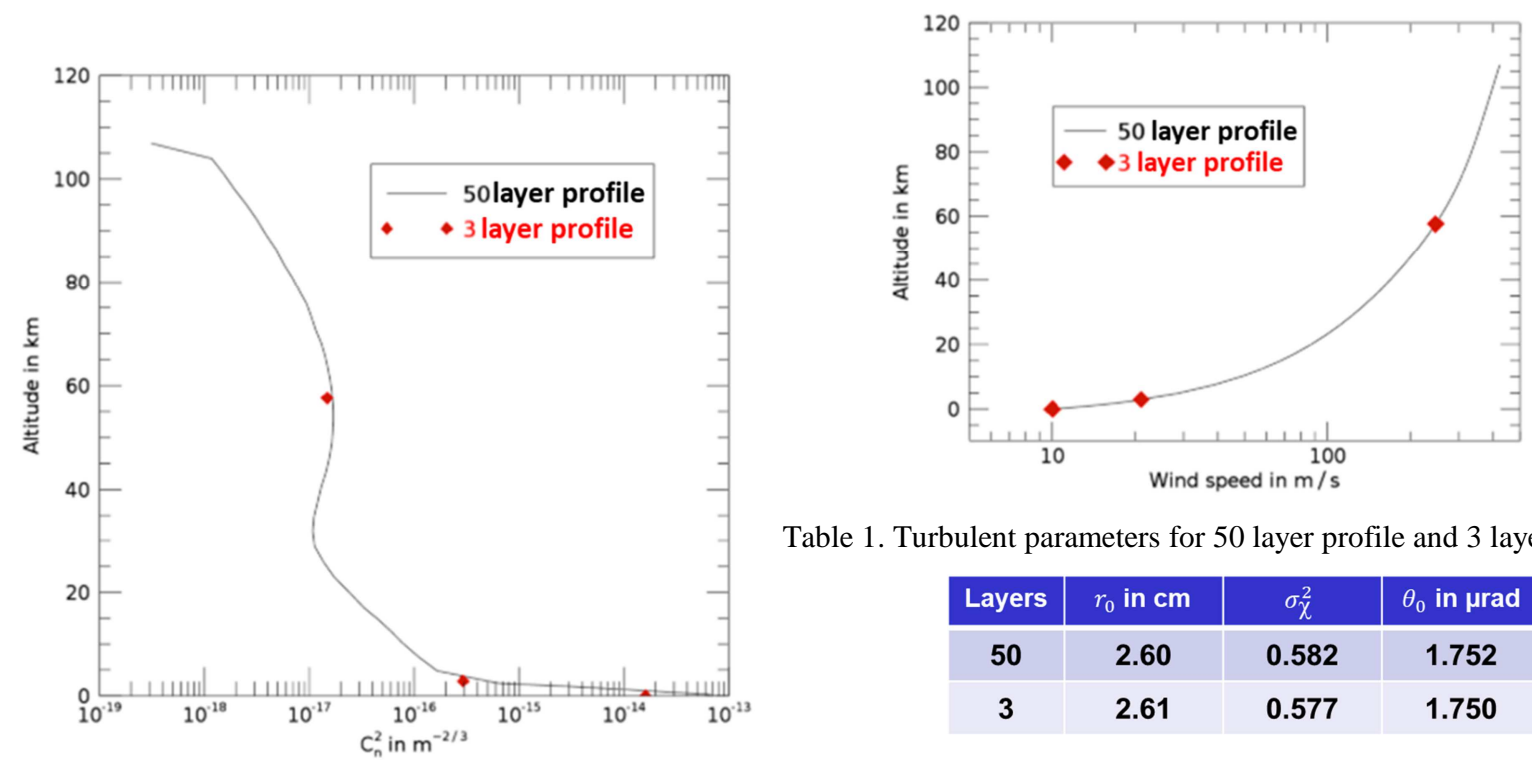

Table 1. Turbulent parameters for 50 layer profile and 3 layer profile

\begin{tabular}{|c|c|c|c|}
\hline Layers & $r_{0}$ in $\mathbf{c m}$ & $\sigma_{\chi}^{2}$ & $\theta_{0}$ in $\boldsymbol{\mu r a d}$ \\
\hline 50 & 2.60 & 0.582 & 1.752 \\
\hline 3 & 2.61 & 0.577 & 1.750 \\
\hline
\end{tabular}

Figure 1. Turbulence profiles, $\boldsymbol{C}_{\boldsymbol{n}}^{2}$ expressed in $\mathrm{m}^{-2 / 3}$ (left) and wind speed in $\mathrm{m} / \mathrm{s}$ taken into account the satellite velocity with the propagation distance, $\mathrm{Z}=0$ at the ground station. 
The turbulence volume is simulated by 3 phase screens. The strength $\left(C_{n}^{2}\right)$ and the location of each screen have to be optimized to be representative of the real turbulence volume. For doing that, the following integrated parameters are considered: the Fried parameter $r_{0}$, the scintillation index or more precisely the variance of the log-amplitude $\left(\sigma_{\chi}^{2}\right)$ and the isoplanatism angle $\theta_{0}$. The reference profile is a Hufnagel/Valley profile with a $C_{n}^{2}$ at ground level equals $10^{-13} \mathrm{~m}^{-2 / 3}$ and a wind speed in altitude (noted vrms) $=21 \mathrm{~m} / \mathrm{s}$. This vertical profile is performed for an elevation angle of $10^{\circ}$ and sampled considering 50 layers. In Figure 1, this profile is plotted in black and the optimised profile defined by 3 layers is represented in red diamond. The 3-layer profile (position and strength of the layer) is obtained by reducing an error criterion taking into account the 3 integrated parameters.

In Table 1, we can check that the 3 integrated parameters are very similar for both profiles. Because we are also interested by simulating temporal effects, we have defined a wind profile taking into account the satellite velocity for both cases (3 and 50 layer profile).

We can conclude that regarding the 3 turbulent parameters, the 3 layer profile is representative of the atmospheric turbulence channel we want to simulate with our platform.

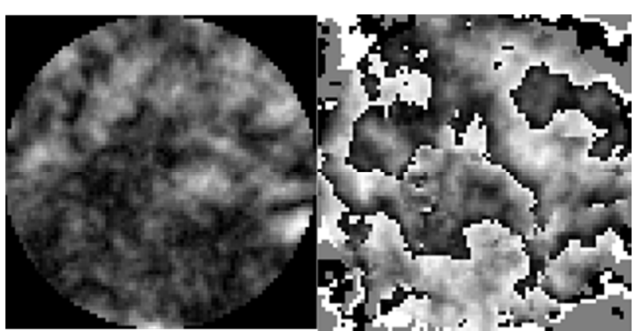

50-layer profile

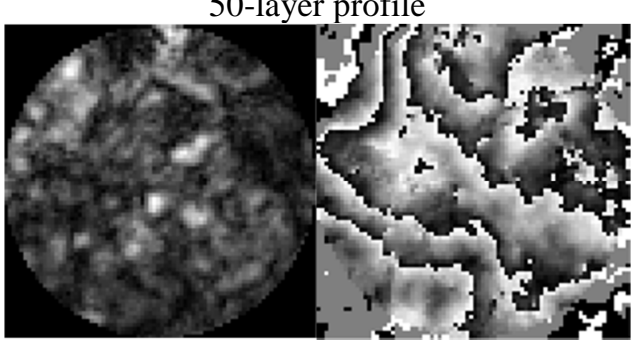

3-layer profile

Amplitude and argument of the propagated beam



Probability density of the flux collected by the whole pupil or by a single pixel



Flux collected by the $40 \mathrm{~cm}$ pupil

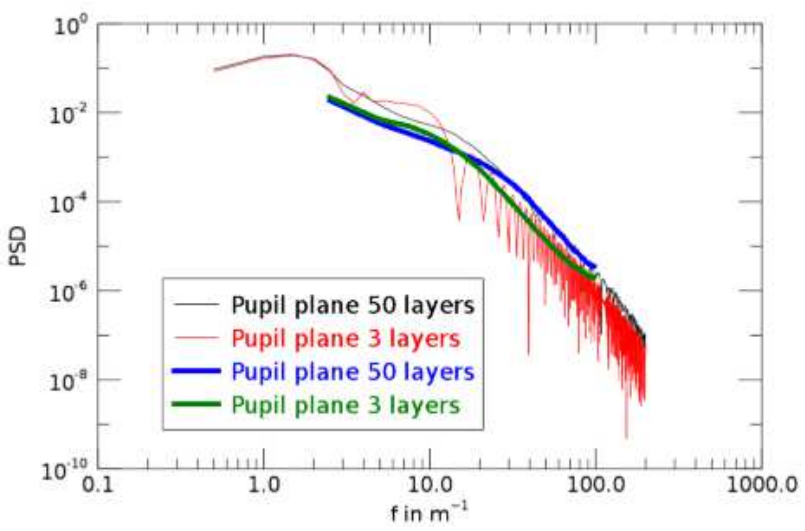

Spatial DSP of the collected flux, red and black theoretical expression, blue and green estimated on simulation results.

Figure 2. Results extracted from the performed simulations and statistical moments of these data, see the legend underneath each figure. 
Then, by wave optics simulation, we have verified that the perturbed beam provided by this 3-layer profile is representative of scintillation and phase effects we want to simulate with the bench. To do this, the statistical moments of the flux collected by a $40 \mathrm{~cm}$ diameter receiver and obtained with both profiles have been compared (see Figure 2) as well as the flux coupled into a single mode fibre (see Figure 3). To check whether the temporal behavior is well simulated we have performed times series and compared their temporal Power Spectral Densities (PSD). As all quantities show, they are in good agreement. In conclusion, the turbulence along the line of sight is well represented by the 3-layer profile regarding scintillation and phase effects.

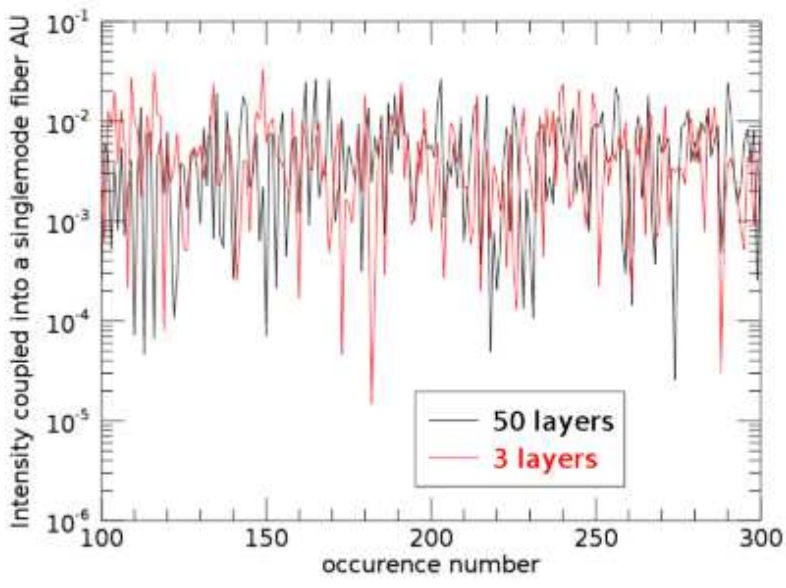

Uncorrelated occurrence of flux coupled into a single mode fiber

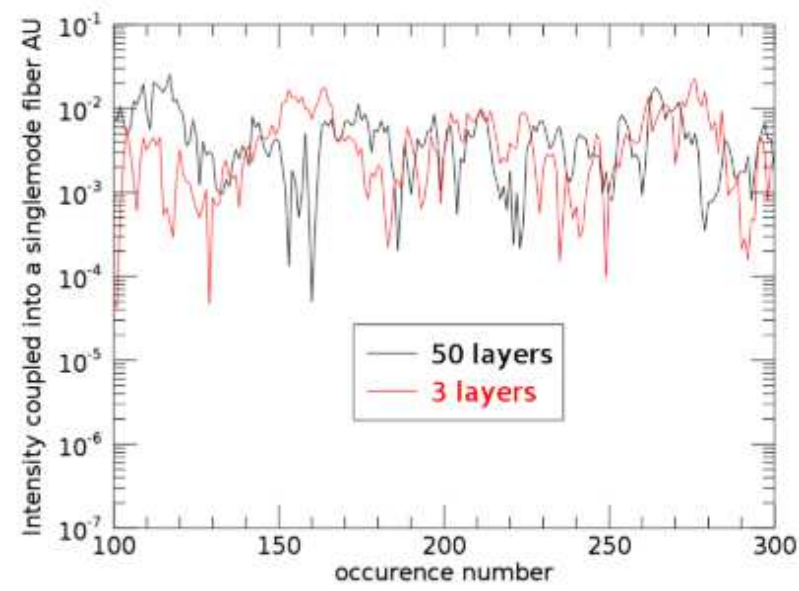

Time series of the flux coupled into a single mode fiber



Probability density of the flux coupled into a single mode fiber and of the Strehl Ratio (SR)

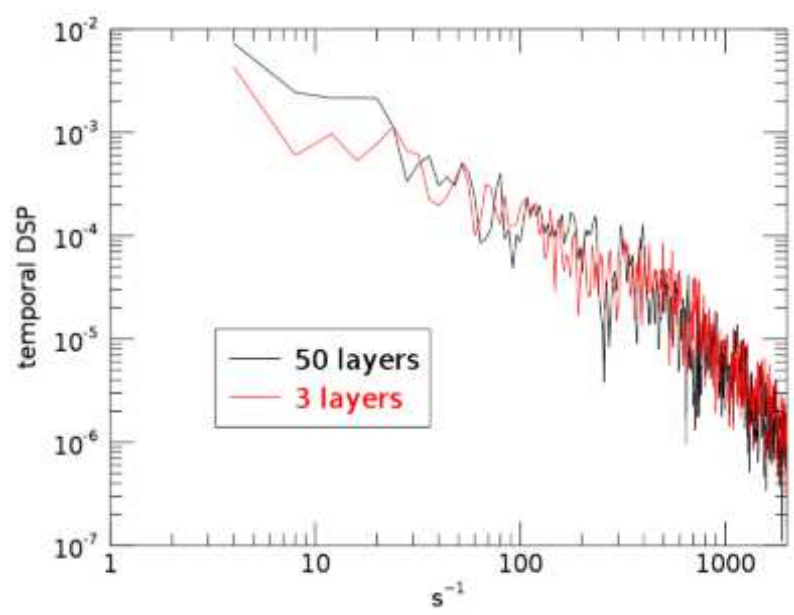

Temporal DSP of the flux coupled into a single mode fiber

Figure 3. Statistical moments of the flux coupled into a single mode fiber in case of uncorrelated simulation (top), in case of temporal correlated simulation (bottom), see the legend underneath each figure.

\subsection{Scaling}

A downscaling of the link geometry was performed. To keep the main relations concerning the laws of wave propagation we have to preserve diffractive effect $(D / \sqrt{\lambda Z})$, phase perturbations $\left(D / r_{0}\right)$ and temporal behavior $\left(V / D / f_{\text {samp }}\right)$ at the level of each layer. $Z$ is the distance between the screen and the entrance pupil of the OGS. $V$ is the velocity of the layer taking into account the satellite velocity; $f_{\text {samp }}$ is the sampling frequency of the AO system loop. For a bench propagation 
distance of less than $1.5 \mathrm{~m}$ and in order to be able to use an AO system operating up to $2 \mathrm{kHz}$, the scaling exercise leads to the features summarized in Table 2.

The number of revolutions per minute (rpm) is estimated for a sampling frequency of $50 \mathrm{~Hz}$. It must be multiplied by 40 for an AO loop running at $2 \mathrm{kHz}$. The pupil diameter is $2 \mathrm{~mm}$ and the propagation distance $1.4 \mathrm{~m}$. The wavelength of the source is $1.55 \mu \mathrm{m}$. The features indicated in the column noted "on-sky" are the values of the operational parameters.

Table 2. Scaling of the link LEO geometry at an elevation of $10^{\circ}$.

\begin{tabular}{|c|c|c|c|}
\hline \multicolumn{2}{|c|}{ Parameters } & On-sky & PICOLO \\
\hline \multicolumn{2}{|c|}{ Propagation distance } & $57 \mathrm{~km}$ & $1.4 \mathrm{~m}$ \\
\hline \multicolumn{2}{|c|}{ Pupil diameter } & $40 \mathrm{~cm}$ & $2 \mathrm{~mm}$ \\
\hline \multicolumn{2}{|l|}{$\lambda$} & $1.55 \mu \mathrm{m}$ & $1.55 \mu \mathrm{m}$ \\
\hline \multicolumn{2}{|l|}{$\mathrm{F}_{\text {samp }} \mathrm{OA}$} & $5 \mathrm{kHz}$ & $50 \mathrm{~Hz}$ (up to $2 \mathrm{kHz}$ ) \\
\hline \multirow{3}{*}{ Layer 1} & Position Z $-D / \sqrt{\lambda Z,}=\propto$ & Pupil (0) & Pupil (0) \\
\hline & $r_{0}(\mathrm{~mm})-\mathrm{D} / \mathrm{r}_{0}=8.9$ & 45 & 0.225 \\
\hline & Wind speed $\mathrm{m} / \mathrm{s}-\mathrm{V} / \mathrm{D} / \mathrm{fech}=0.005$ & 10 & 0.02 \\
\hline \multirow{3}{*}{ Layer 2} & Position Z $-D / \sqrt{\lambda Z,}=6$ & $2.88 \mathrm{~km}$ & $0.072 \mathrm{~m}$ \\
\hline & $r_{0}(\mathrm{~mm})-\mathrm{D} / \mathrm{r}_{0}=6.7$ & 59 & 0.295 \\
\hline & Wind speed $\mathrm{m} / \mathrm{s}-\mathrm{V} / \mathrm{D} / \mathrm{fech}=0.013$ & 21.1 & 0.052 \\
\hline \multirow{3}{*}{ Layer 3} & Position $Z-D / \sqrt{\lambda Z,}=1.34$ & $57.8 \mathrm{~km}$ & $1.4 \mathrm{~m}$ \\
\hline & $r_{0}(\mathrm{~mm}) \mathrm{D} / \mathrm{r}_{0}=1.2$ & 345 & 1.7 \\
\hline & Wind speed $\mathrm{m} / \mathrm{s}-\mathrm{V} / \mathrm{D} / \mathrm{fech}=0.125$ & 247 & 0.5 \\
\hline
\end{tabular}

\subsection{Design}

The set-up is presented in Figure 4. The source is a collimated laser emitting at $1.55 \mu \mathrm{m}$. A first phase screen (noted PS3) is close to the source at the highest altitude, the other two (PS1 and PS2) are close to the entrance pupil which is marked by the central violet arrow. Two other planes are conjugated with the entrance pupil (see 2 other violet arrows): one on the SLM and the other one on the mirror of the output periscope. A second path (in blue) is dedicated to the analysis of the perturbation. Either the focal plane or the pupil plane can be recorded on the camera thanks to a foldable lens.



Figure 4. Top (left) and 3D (right) views of the set-up.

The position of the phase screens can be modified to simulate other turbulence profiles. The revolution speed of each phase screen can also be adapted to be representative of different wind speed conditions.

The advantage of introducing a SLM in a plane conjugated of the entrance pupil is to be able to add known turbulence burst, a pre-compensation or the addition of known aberrations on the propagation channel at low frequency enabling by the use of this kind of device. 


\section{INTEGRATION AND TESTS}

\subsection{Phase screen characterization}

The transmissive phase screens (PS) have been realized by SILIOS Technologies (located in the South of France). We have provided to them numerical phase screens following Von Karman spectrum. An outer scale $L_{0}$ is taken into account which equals $1.3 \mathrm{~m}$ for both screens close to the ground and $4 \mathrm{~m}$ for the highest altitude one. The numerical phase cartography of the less turbulent PS (PS3 highest altitude) is given in Figure 5 (left). The theoretical variance of Zernike modes (considering a Kolmogorov spectrum, with an infinite outer scale) sorted by radial orders is compared to those evaluated on the simulated phase screen. The difference at low radial orders $(n=1)$ is due to the fact that $\mathrm{L}_{0}$ is considered as infinite in Kolmogorov spectrum and the real screens an outer scale is taken into account. This specification has been used to characterize and accept the manufactured phase screens.

The phase perturbation produced by the PS has been measured with a Shack-Hartmann wavefront sensor. Experimental Zernike variances are very similar to those expected.

Each phase screen is mounted in a rotating holder which can spin up to $300 \mathrm{rpm}$ (revolution per minute). It is therefore possible to test an $\mathrm{AO}$ system operating with a $2 \mathrm{kHz}$ loop.

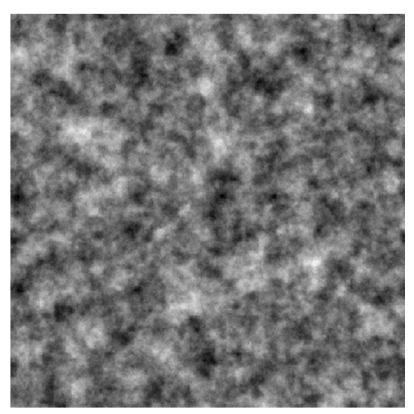

Numerical Phase Screen (PS3), $\mathbf{r}_{0}=35 \mathrm{~cm}$ (on the sky)

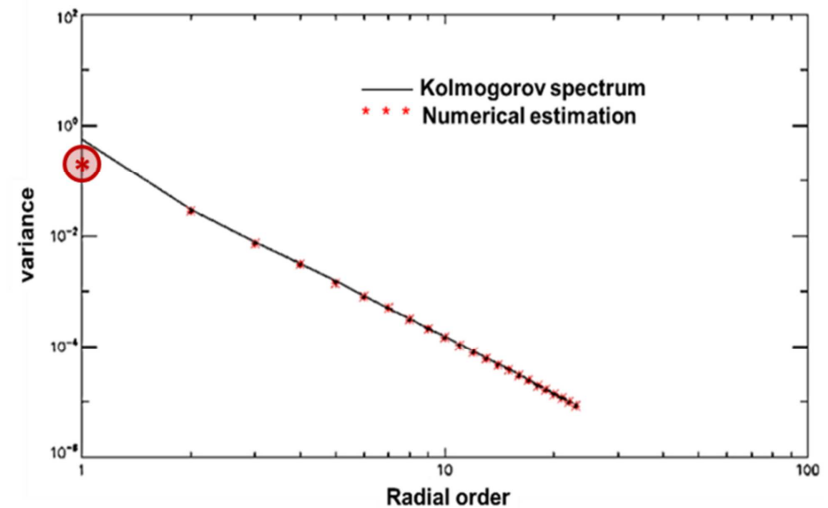

Variance of the Zernike modes

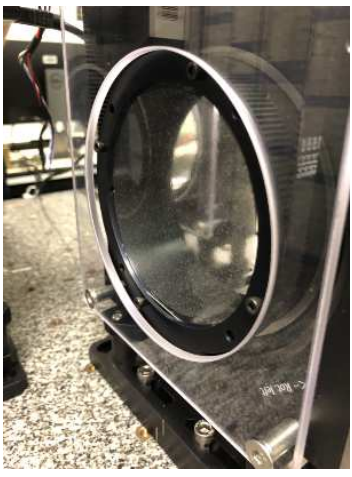

Phase screen in its rotating holder.

Figure 5. Phase Screen: features and picture of the mounted phase screen.

\subsection{Preliminary results}

For the moment only a qualification of the effect induced by the phase screens has been considered. An illustration of the results obtained is shown in Figure 6. When using a strongly turbulent PS (PS1 and PS2), the effect in the focal plane is significant: the point spread Function (PSF) is widely spread compared to the non-turbulent spot and presents several speckles. With PS3, the PSF is very similar to the non-turbulent one but is wandering when the screen is rotated to simulate temporal effects.

In the pupil plane, the illumination is not homogeneous when PS2 or PS3 are positioned in their respective planes as can be seen in Figure 6. The size of the speckles is smaller when the phase screen is closer to the entrance pupil which is expected because it is proportional to $\sqrt{\lambda \mathrm{Z}}$. 
With PS2

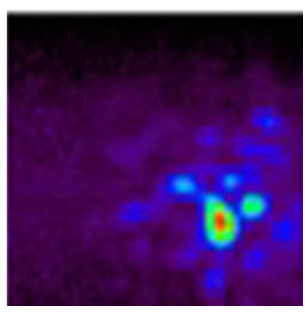

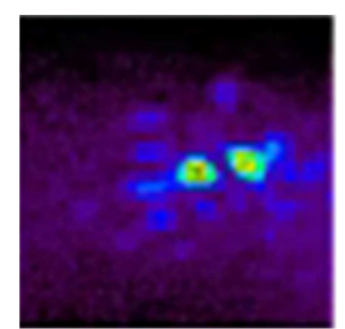

In the focal plane (PSF)

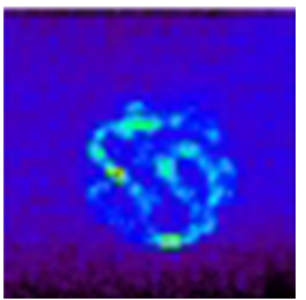

In the pupil plane
With PS3
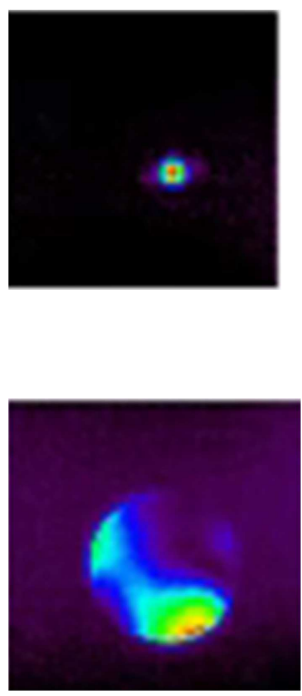

Figure 6. Top: Focal plane images obtained in the presence of the different phase screens. Bottom : pupil plane images.

\section{CONCLUSIONS}

The characterization of the bench is ongoing. The experimental results will be compared with the numerical results taking into account the same criteria as those used when selecting number and position of phase screens.

This bench delivers a turbulence-perturbed laser beam representative of what can be expected in the case of a downlink between a LEO satellite and the ground at $10^{\circ}$ elevation. Link with GEO satellites can be simulated by changing the phase screens. Its ability to be representative of an uplink is under evaluation, in particular with regard to the PointAhead angle (anisoplanatism effect).

It can provide long time series of coupled flux into a single mode fiber with or without an AO system.

It constitutes a test platform for future AO systems (wavefront measurement and control laws) for the management of strong perturbations (scintillation, unstationnarities, ...). It enables a "client" system to receive a disturbed beam disturbed at its entrance pupil in order to test new AO concepts, to integrate and to validate them before on-sky campaigns.

\section{REFERENCES}

[1] Brady, A., Eberhardt, R., Tünnermann, A., and Reinlein, C, "Demonstration of Adaptive Optical PreCompensation in Horizontal Tests", IEEE International Conference on Space Optical Systems and Applications (ICSOS), 1-5 (2019).

[2] Kudielka, K., E. Fischer, and T. Dreischer, "Numerical prediction and experimental validation of irradiance fluctuations in a pre-compensated optical feeder link", International Conference on Space Optic (ICSO 2018). Vol. 11180, International Society for Optics and Photonics (2019).

[3] Qu, Zhen, and Ivan B. Djordjevic, "Approaching terabit optical transmission over strong atmospheric turbulence channels", 18th International Conference on Transparent Optical Networks (ICTON), IEEE (2016). 